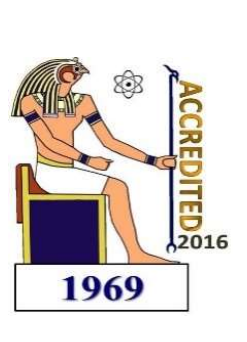

Delta Journal of Science

Available online at

https://djs.journals.ekb.eg/

\title{
Synthesis and Characterization of Some Quinazolone Derivatives
}

\author{
A. A. El-Barbary A. M. Sharaf \\ Chemistry Department, Faculty of Science, Tanta University, Egypt \\ Corresponding author e-mail: aeelbarbary @hotmail.com
}

\begin{abstract}
Reaction of compound 1 with 2,3,4,6-tetra-O-acetyl- $\alpha$-D-glucopyranosyl bromide (ABG) afforded 2. Deplocking of 2 with sodium methoxide gave 1. Alkylation of 1 led to the formation of 3a-c. Fusion of 1 with ethyl chloroformate and/or ethyl chloroacetate gave 4a,b. Similarly, boiling of 1 with ethyl chloroacetoacetate gave 5 . Refluxing of 1 with diphenyl diazomethane gave 6. Compound 7 reacted with some aromatic aldehydes and triethyland/or triphenyl- phosphite in glacial acetic acid to furnish the amino phosphonates 8a-d. Treatment of 7 with 4 aminobenzoic acid gave 9. Boiling 7 and triethylorthoformate in glacial acetic acid gave 10. Condensation of 7 with 4benzylidene-2-phenyloxazol-5(4H)-one furnished 11. Reaction of 7 with 4-chlorophenyl isocyanate and/or phenyl isothiocyanate in boiling anhydrous pyridine gave 12 and/or 14. Refluxing 7 with chloroacetaldehyde in ethanol yielded 15. Fusion of 7 with some sultones afforded the corresponding sultams $18 \mathrm{a}, \mathrm{b}$. All the new compounds were tested for their potential antibacterial activates and the results indicated that some of them showed activity against different types of bacteria.
\end{abstract}

Key words: quinazolonones, alkylation, aminophosphonates, sultams, biological activity.

\section{Introduction:}

As our research group for some time ago is involved in the chemistry of quinazolone derivatives ${ }^{1-3}$ due to their importance in biological activity as antihypertensive, ${ }^{4}$ antifibrillatory, choleretic, antiphlogistic, ${ }^{5}$ antimitotic anticancer, ${ }^{6}$ antifungal ${ }^{7}, 8$ and anticonvulsant agents. $^{9}$ Quinazolinones were reported to possess diverse pharmacological activities such as CNS depressant, ${ }^{10}$ hypnotic, antiinflamatory, ${ }^{11}$ antitumor, ${ }^{12}$ muscle relaxants ${ }^{13}$ and for their antineoplastic activity. ${ }^{14}$ So, it is our goal to extend our study in this area to explore the reactivity of 3-phenylquinazoline-2,4(1H,3H)-dithione (1) ${ }^{15}$ and 3-amino-6,8-dibromo-2-thioxo-2,3-dihydro- $1 H$ quinazolin-4-one (7) towards different reagents to synthesize some new derivatives for testing their biological activities.

\section{RESULTS AND DISCUSSION}

Reaction of 3-phenylquinazoline-2,4(1H,3H)dithione (1) ${ }^{15}$ with (2,3,4,6-tetra- $O$-acetyl- $\alpha$-D-glucopyranosyl)bromide $(\square-\mathrm{ABG})$ in the presence of triethyl amine in DMF at room temperature afforded the corresponding $\mathrm{S}$ nucleoside 2. Deblocking of $\mathbf{2}$ using sodium methoxide at r.t. yielded the starting aglycone, ${ }^{1,16}$ and not the desired deblocked compound 3-phenyl-2-((2R,3S,4R,5R)-3,4,5trihydroxy-6-(hydroxymethyl)tetrahydro-2H-pyran-2ylthio)quinazoline-4(3H)-thione (2a). Its IR spectrum showed the $\mathrm{C}=\mathrm{O}$ at $1748 \mathrm{~cm}^{-1}$ and its ${ }^{1} \mathrm{H}-\mathrm{NMR}$ spectrum showed a singlet $\left(12 \mathrm{H}, 4 \mathrm{CH}_{3}\right)$ at $1.96 \mathrm{ppm}$ and its $\mathrm{MS}$ showed the EI $\left(\mathrm{M}^{+}\right)$at 600.25 . S-alkylation of compound 1 could be achieved by its treatment with alkylating agents namely: methyl iodide, benzyl chloride and /or phenacyl 
chloride at room temperature to yield the corresponding 2alkylthio derivatives 3a-c, respectively (Scheme 2). The ${ }^{1} \mathrm{H}-\mathrm{NMR}$ spectrum of compound $\mathbf{3 a}$ showed a singlet $\left(\mathrm{CH}_{3}\right)$ at $2.75 \mathrm{ppm}$

Fusion of compound 1 with ethyl chloroformate and / or ethyl chloroacetate yielded the corresponding 2-alkylthio derivatives (4a, b) (Scheme 2). The IR spectrum of compound 4 a showed the carbonyl $(\mathrm{C}=\mathrm{O})$ ester at 1760 $\mathrm{cm}^{-1}$.

By the same manner refluxing compound 1 with ethyl chloroacetoacetate in methanol for $4 \mathrm{hr}$ afforded ethyl-3oxo-2-(3-phenyl-4-thioxo-1,2,3,4-tetrahydro- quinazolin2-ylthio) butanoate (5) (Scheme 2). Its IR spectrum showed the $(\mathrm{C}=\mathrm{O})$ ketone at 1665 and the $(\mathrm{CO})$ ester at $1742 \mathrm{~cm}^{-1}$, its ${ }^{1} \mathrm{H}-\mathrm{NMR}$ spectrum showed a triblet $3 \mathrm{H}$ of $\left(\mathrm{CH}_{3}\right)$ at 1.64 , quartet $2 \mathrm{H}\left(\mathrm{CH}_{2}\right)$ at 3.41 and a singlet $(\mathrm{CH})$ at $3.95 \mathrm{ppm}$. Refluxing of compound 1 with diphenyl diazomethane (prepared according to known method $)^{17}$ in anhydrous benzene for $6 \mathrm{hr}$ gave 2(benzhydrylthio)-3-phenyl quinazoline-4(3H)-thione (6) as the sole product (tlc) and not $6 \mathbf{a}$ or $6 \mathbf{b}$ (Scheme 2 ). The structures $6 \mathbf{a}$ and $6 \mathbf{b}$ were ruled out based on different spectroscopic data. The IR spectrum of $\mathbf{6}$ showed the disappearance of $\mathrm{SH}$ at $1653 \mathrm{~cm}^{-1}$, while appearance of $\mathrm{SCH}$ at $2927 \mathrm{~cm}^{-1}$ and its ${ }^{1} \mathrm{H}-\mathrm{NMR}$ spectrum showed a singlet $(\mathrm{CH})$ at $3.51 \mathrm{ppm}$. and its MS showed the $\mathrm{m} / \mathrm{z}\left(\mathrm{M}^{+}\right.$ ) at 436.59 .

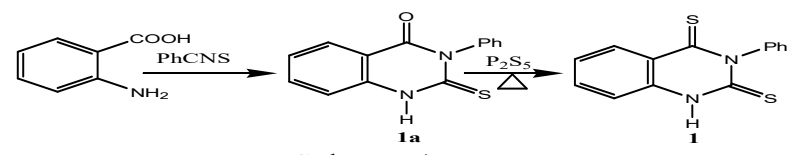

Scheme 1

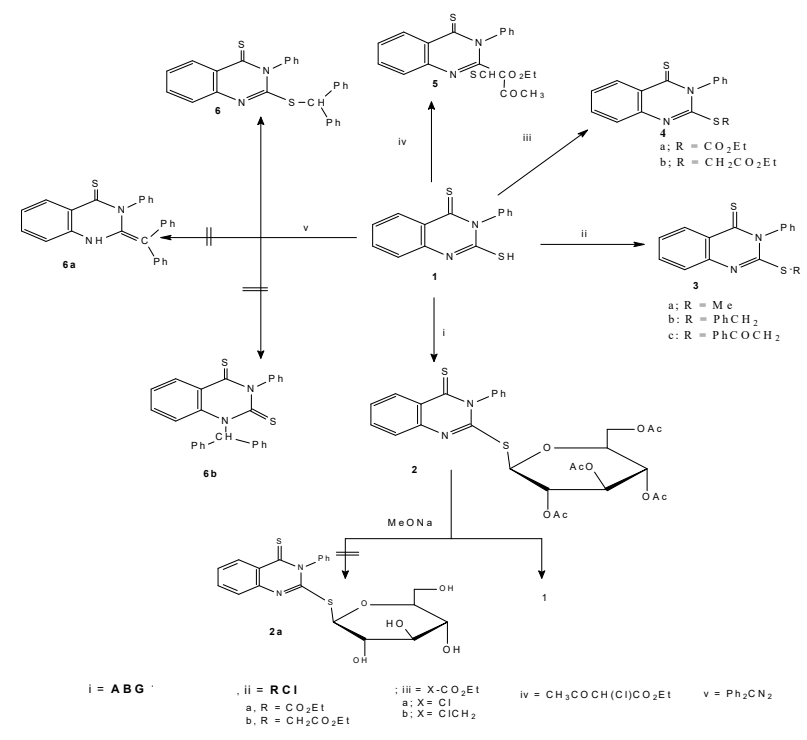

Scheme 2

In our earlier work ${ }^{1}$ we found that 3 -amino-6,8-dibromo2-thioxo-2,3-dihydro-1H-quinazolin-4-one (7) was a versatile compound due to its biological activity as Accaros: Spider mite (Tetranychus- urticae),(Koch), Fungicides (Rhizoctonia solani, Fusarium oxysporium,
Fusarium solani, Verticillium dahliae and Verticillium sulphurellium)and Bactericides (Psedomonas solaniserum, Erwinia carotovora and Ralstonia salanceanum). ${ }^{1}$

So, we found it is worthy to extend our study on the chemistry of compound 7 to get new derivatives of expected biological activity.

Accordingly, Compound 7 reacted with a mixture of aromatic aldehydes(benzaldehydeand4chlorobanzaldehyde)andtriethyl-and/or triphenylphosphite in glacial acetic acid at $100^{\circ} \mathrm{C}$ for $4-6 \mathrm{hr}$ (tlc) to furnish the amino phosphonates (8a-d), respectively. The IR spectrum of compound 8a showed the $(\mathrm{CH})$ at 2849 and $(\mathrm{NH})$ at $3300 \mathrm{~cm}^{-1}$. The ${ }^{1} \mathrm{H}-\mathrm{NMR}$ spectrum of compound $\mathbf{8 b}$ showed the triplet $\left(2 \mathrm{CH}_{3}\right)$ at $1.20,(\mathrm{CH})$ at 3.94 and $\left(\mathrm{NH}_{\text {acyclic }}\right)$ at 2.51 ppm.. The ${ }^{13} \mathrm{C}-\mathrm{NMR}$ spectrum of compound 8c showed the $(\mathrm{CH})$ at 53.55 , (P-O-C) at 154.33 and $(\mathrm{C}=\mathrm{O})$ at $169.22 \mathrm{ppm}$.

Treatment of compound 7 with 4-aminobenzoic acid in boiling phosphorus oxychloride for $8 \mathrm{hr}$ gave 2-(4aminophenyl)-7,9-dibromo-10,10a-dihydro-

$[1,3,4]$ thiadiazolo[2,3-b]quinazolin-5-one (9) through the elimination of two molecules of water. Its ${ }^{13} \mathrm{C}-\mathrm{NMR}$ spectrum showed the $(\mathrm{C}=\mathrm{C})$ at $153.15,(\mathrm{C}=\mathrm{O})$ at 164.32 and $(\mathrm{C}=\mathrm{N})$ at $170,31 \mathrm{ppm}$.

Boiling amixture of compound 7 and triethylorthoformate in glacial acetic acid for $3 \mathrm{hr}$ (tlc) gave ethylN-6,8dibromo-4-oxo-2-thioxo-1,2-dihydroquinazolin-3(4H)ylformimidate(10).ItsIR spectrum showed the $(\mathrm{CH})$ at $2950 \mathrm{~cm}^{-1}$ and Its ${ }^{13} \mathrm{C}$-NMR spectrum showed the $\left(\mathrm{CH}_{3}\right)$ at 14.19 and $\left(\mathrm{CH}_{2}\right)$ at $61.52 \mathrm{ppm}$.

Condensation of compound 7 with 4-benzylidene-2phenyloxazol-5(4H)-one in boiling glacial acetic acid furnished3-(4-benzylidene-5-oxo-2-phenyl-4,5dihydroimidazol-1-yl)-6,8-dibromo-2-thioxo-1,2,3,8atetrahydroquinazolin-4(4aH)-one (11). Its IR spectrum showed a sharp signal at $2970 \mathrm{~cm}^{-1}$ for $\left(\mathrm{CH}_{\text {ar. }}\right)$ and its $\mathrm{MS}$ spectrum showed the $\mathrm{m} / \mathrm{z}$ at $(581,14 \%),\left(\mathrm{M}^{+}, \mathrm{C}_{24} \mathrm{H}_{15} \mathrm{Br}_{2} \mathrm{~N}_{4} \mathrm{O}_{2} \mathrm{~S}_{2}\right)$.

Reaction of compound 7 with 4-chlorophenyl isocyanate and/or phenyl isothiocyanate in boiling anhydrous pyridine for 4-5 hr gave 1-(4-chlorophenyl)-3-(6,8dibromo-4-oxo-2-thioxo-1,2-dihydroquinazolin-3(4H)-

yl)urea (12) and/or 7,9-dibromo-2-(phenylimino)-2,3dihydro-

[1,3,4]thiadiazolo[2,3-b]quina-zolin-5-one(14), respectiveely. The IR spectrum of compound 12 showed the $(\mathrm{C}=\mathrm{O}-$ $\mathrm{NH})$ at $1660,(\mathrm{CH})$ at $2961 \mathrm{~cm}^{-1}$. The ${ }^{13} \mathrm{C}-\mathrm{NMR}$ spectrum of compound 14 showed the $(\mathrm{C}=\mathrm{N})$ at $147.17,(\mathrm{C}=\mathrm{O})$ at 156.86 and $(\mathrm{C}-\mathrm{S})$ at $160.66 \mathrm{ppm}$. 


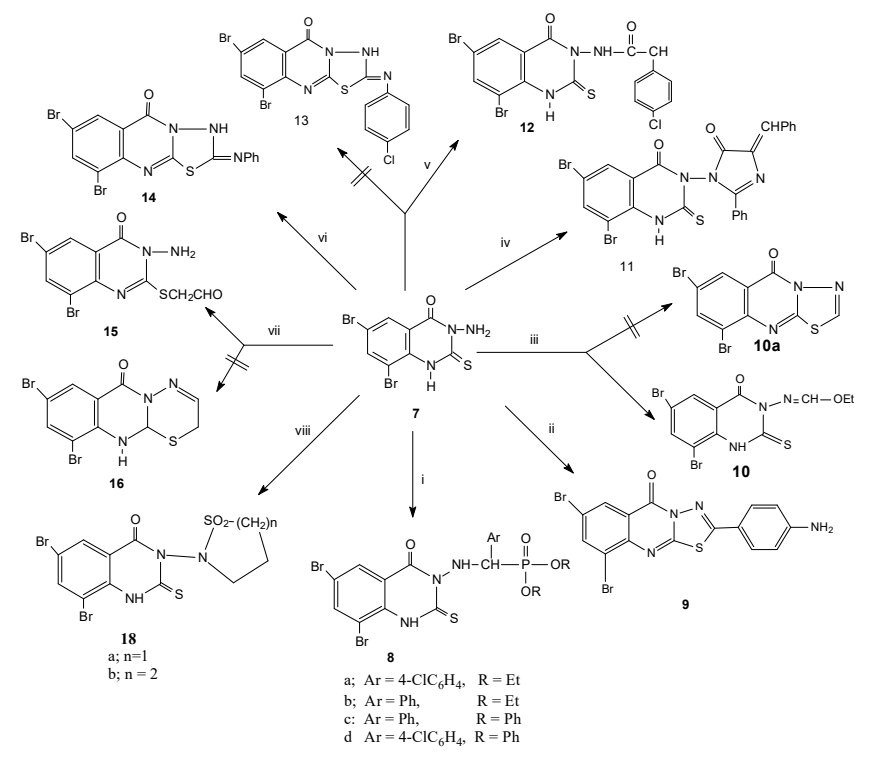

$\mathrm{i}=\mathrm{ArCHO}, \mathrm{P}(\mathrm{OR})_{3}, \mathrm{ii}=4-\mathrm{NH}_{2} \mathrm{C}_{6} \mathrm{H}_{4} \mathrm{CO}_{2} \mathrm{H} ; \mathrm{iii}=-\mathrm{CH}(\mathrm{OEt})_{3}$

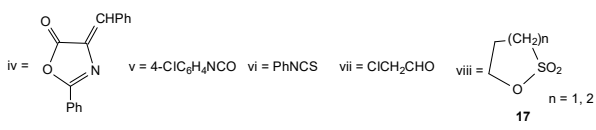

Scheme 3

Refluxing a mixture of compound 7 and chloroacetaldehyde in ethanol for $3 \mathrm{hr}$ yielded 2-(3amino-6,8-dibromo-4-oxo-1,2,3,4-tetrahydroquinazolin-2ylthio)acetalde-hyde (15). Its IR spectrum showed the disappearance of $\mathrm{C}=\mathrm{S}$ at $1120 \mathrm{~cm}^{-1}$, while appearance of $\mathrm{C}=\mathrm{N}$ at $1560 \mathrm{~cm}^{-1}$ and its ${ }^{1} \mathrm{H}-\mathrm{NMR}$ spectrum showed signal $\left(2 \mathrm{H}, \mathrm{CH}_{2}\right)$ at 4.43 and a single $(1 \mathrm{H}, \mathrm{CHO})$ at 9.70 ppm.

Fusion of compound 7 with 1,3-propane-and/or 1,4butane-sultone at $180{ }^{\circ} \mathrm{C}$ for $9-11 \mathrm{hr}$ afforded the corresponding sultams: 6,8-dibromo-2-thioxo-2,3dihydro-quinazolinoyl-propane-1,3-sultam (18a) and 6,8dibromo-2-thioxo-2,3-dihydroquinazolinoyl-butane-1,4sultam (18b), respectively (Scheme 3). The ${ }^{1} \mathrm{H}-\mathrm{NMR}$ spectrum of compound $\mathbf{1 8 a}$ showed the $\left(\mathrm{m}, 2 \mathrm{H}, \mathrm{CH}_{2}\right)$ at $1.20,\left(\mathrm{~d}, 2 \mathrm{H}, \mathrm{CH}_{2}\right)$ at 3.01 and $\left(\mathrm{d}, 2 \mathrm{H}, \mathrm{CH}_{2}\right)$ at $3.20 \mathrm{ppm}$. The IR spectrum of compound $18 \mathbf{b}$ showed the $\left(\mathrm{SO}_{2}\right)$ at $1156,1350 \mathrm{~cm}^{-1}$ and $(\mathrm{C}=\mathrm{S})$ at $1120 \mathrm{~cm}^{-1}$.

\section{EXPERIMENTAL}

All melting points were uncorrected and performed by the open capillary melting point apparatus. Microanalyses were performed by Microanalysis Unit, Faculty of Science, Cairo University and Microanalysis Unit, Central Laboratory, Tanta University. IR spectra recorded with a Perkin-Elmer 1720 spectrometer. The NMR spectra were recorded on a Brüker AC $250 \mathrm{FT}$ NMR spectrometer at $250 \mathrm{MHz}$ for ${ }^{1} \mathrm{H}$ and $62.9 \mathrm{MHz}$ for ${ }^{13} \mathrm{C}$, Varian UNITY $500 \mathrm{NMR}$ spectrometer at $500 \mathrm{MHz}$ for ${ }^{1} \mathrm{H}$ or $125.7 \mathrm{MHz}$ for ${ }^{13} \mathrm{C}$, Bruker $200 \mathrm{MHz}$ And Bruker $90 \mathrm{MHz}$ spectrometer using TMS as an internal standard DMSO as a solvent. Chemical shifts $(\delta)$ are reported in parts per million (ppm) and signals are expressed as s (singlet), $d$ (doublet), $\mathrm{t}$ (triplet), q (quartet), $\mathrm{m}$ (multiplet) or br (broad). Mass spectra (MS) were recorded using electron ionization (E.I.) on a Varian Mat 311A spectrometer.

Compounds $1 a^{15}, \mathbf{1}^{15}$ and $7^{1}$ were prepared according to recommended methods.

Coupling of 1 with 2,3,4,6-tetra-O-acetyl- $\alpha$-Dglucopyranosyl bromide (ABG). Formation of 2.

A solution of compound $\mathbf{1}(0.27 \mathrm{~g}, 0.001 \mathrm{~mol})$ was dissolved in a mixture of triethyl amine $(2 \mathrm{ml})$ and $\mathrm{DMF}$ $(15 \mathrm{ml})$ and added to a solution of ABG $(0.41 \mathrm{~g}, 0.001$ $\mathrm{mol})$. The reaction mixture was stirred at $\mathrm{r}$. $\mathrm{t}$. for $4 \mathrm{hr}$ (tlc). The reaction mixture was pourd onto cold water, the solid formed was filtered off, washed with water, dried, and recrystallized from ethanol to afford $2^{16}$.

\section{2-(2,3,4,6-Tetra-O-acetyl- $\beta$-D-glucopyranosyl-thio)-3-} phenyl-2,3dihydro-quinazoline-4(1H)-thione (2)

IR: $v\left(\mathrm{~cm}^{-1}\right) 1227(\mathrm{C}=\mathrm{S}$ cyclic $), 1610(\mathrm{C}=\mathrm{N}), 1748(\mathrm{C}=\mathrm{O}$, acetyl), $2925(\mathrm{CH}) ;{ }^{1} \mathbf{H}-\mathbf{N M R}\left(\mathrm{DMSO}-d_{6}\right): \delta 1.96(\mathrm{~s}, 12 \mathrm{H}$, $\left.4 \mathrm{CH}_{3}\right), 4.01-4.30\left(\mathrm{~s}, 2 \mathrm{H}, \mathrm{CH}_{2}\right), 4.96(\mathrm{dd}, 1 \mathrm{H}, J=5.63 \mathrm{~Hz}$, H-5 ), 5.10 (t, 1H, $\left.J=3.04 \mathrm{~Hz}, \mathrm{H}-4^{\prime}\right), 5.36(\mathrm{dd}, 1 \mathrm{H}, J=$ $\left.4.98 \mathrm{~Hz}, \mathrm{H}-3^{`}\right), 5.58$ (dd, $\left.1 \mathrm{H}, J=3.85 \mathrm{~Hz}, \mathrm{H}-2^{`}\right), 5.97$ (dd, $\left.1 \mathrm{H}, J=4.16, \mathrm{H}^{-1}\right), 7.39-8.55\left(\mathrm{~m}, 9 \mathrm{H}, \mathrm{H}_{\mathrm{ar}}\right) \mathrm{ppm} ;{ }^{13} \mathrm{C}-$ NMR (DMSO-d $\left.d_{6}\right): \delta 20,19\left(\mathrm{CH}_{3}, \mathrm{C}^{\prime} 6\right), 20,35\left(\mathrm{CH}_{3}, \mathrm{C}^{\prime} 4\right)$, $20.36\left(\mathrm{CH}_{3}, \mathrm{C}^{\prime} 2\right), 20.38\left(\mathrm{CH}_{3}, \mathrm{C}^{\prime} 3\right), 61.65,67.94,68.50$, 73.01, 75.06, 81.90 (C'6, C'4, C'2, C'3, C'5, C'1 1 anomeric), $127.19,128.84,129.04,129.84,130.20\left(\mathrm{C}_{\text {ar. }}\right), 153.51$ $\left(\mathrm{C}=\mathrm{N}_{\text {cyclic }}\right), 169.08,169.20,169.20,169.72,169.87(\mathrm{C}=$ $\mathrm{O}$, acetyl $), 189.08\left(\mathrm{C}=\mathrm{S}_{\text {cyclic }}\right) \mathrm{ppm}$.; MS (EI) $\mathrm{m} / \mathrm{z}=600.25$ $\left(\mathrm{M}^{+}, \mathrm{C}_{28} \mathrm{H}_{28} \mathrm{~N}_{2} \mathrm{O}_{9} \mathrm{~S}_{2}, 100 \%\right)$.

\section{Deblocking of Compound 2.}

Compound 2 (0.60 g, $0.001 \mathrm{~mol})$ was dissolved in $(20 \mathrm{ml})$ methanol and two drops of sodium methoxide solution $(0.001 \mathrm{~N})$ were added. The reaction mixture was left at room temperature for $4 \mathrm{hr}$ (tlc). The solvent was evaporated under vacuum and the residual solid was dissolved in water neutralized with dill $\mathrm{HCl}$. The solid formed was filtered off, washed with water, dried, recrystallized from ethanol to give the starting material $\mathbf{1}^{16}$. Yield $0.93 \mathrm{~g}(62 \%)$; m.p. $180-182{ }^{\circ} \mathrm{C}$. mp and mixed $\mathrm{mp}$ of the product with authentic sample of $\mathbf{1}$ gave no depression.

\section{Alkylation of 1. Formation of 3a-c.}

Compound 1 (0.81g, $0.003 \mathrm{~mol})$ was dissolved in methanol $(20 \mathrm{ml})$ and sodium hydroxide $(0.5 \mathrm{~g})$. The appropriate alkyl halide $(0.003 \mathrm{~mol})$ was added, and the reaction mixture was stirred at r.t. for $4-5 \mathrm{hr}$ (tlc). The solid product formed was filtered off, recrystallized from ethanol, filtered, and dried to afford 3a-c.Table 1and Scheme 2.

\section{2-(Methylthio)-3-phenylquinazoline-4(3H)-thione (3a).}

IR: $v\left(\mathrm{~cm}^{-1}\right) 1188\left(\mathrm{C}=\mathrm{S}_{\text {cyclic }}\right), 1665(\mathrm{C}=\mathrm{N}), 2925(\mathrm{CH}) ;{ }^{1} \mathbf{H}-$ NMR (DMSO-d $d_{6}$ (fig. 9): $\delta 2.75\left(\mathrm{~s}, 3 \mathrm{H}, \mathrm{CH}_{3}\right), 6.88-$ $7.88\left(\mathrm{~m}, 9 \mathrm{H}, \mathrm{H}_{\mathrm{ar}}\right) \mathrm{ppm} ;{ }^{13} \mathbf{C}-\mathbf{N M R}\left(\mathrm{DMSO}-d_{6}\right): \delta 14,16$ $\left(\mathrm{CH}_{3}\right), 122.09,124.14,126.18,128.91,136.81,143.12$ 
and $149.12\left(\mathrm{C}_{\text {ar. }}\right), 163.26\left(\mathrm{C}=\mathrm{N}_{\text {cyclic }}\right), 179.12(\mathrm{C}=\mathrm{S}) \mathrm{ppm}$; MS (EI) $\mathrm{m} / \mathrm{z}=284,05\left(\mathrm{M}^{+}, \mathrm{C}_{15} \mathrm{H}_{12} \mathrm{~N}_{2} \mathrm{~S}_{2}, 1.7 \%\right)$.

\section{2-(Benzylthio)-3-phenylquinazoline-4(3H)-thione (3b).}

IR: $v(\mathrm{~cm}-1) 1200\left(\mathrm{C}=\mathrm{S}_{\text {cyclic }}\right), 1690(\mathrm{C}=\mathrm{N}), 2875\left(\mathrm{CH}_{\text {aliph. }}\right)$, $3037\left(\mathrm{CH}_{\text {ar. }}\right) ;{ }^{1} \mathrm{H}-\mathrm{NMR}$ (DMSO-do): $\delta 4.51\left(\mathrm{~s}, 2 \mathrm{H}, \mathrm{CH}_{2}\right)$, $7.25-8.57\left(\mathrm{~m}, 14 \mathrm{H}, \mathrm{H}_{\mathrm{ar}}\right)$ ppm; ${ }^{13} \mathbf{C}-\mathbf{N M R}$ (DMSO-d6) (fig. 14): $\delta 39.29\left(\mathrm{CH}_{2}\right), 126.83,127.38,128.46,129.02$, $129.41,129.84,129.94$ and $135.45\left(\mathrm{C}_{\mathrm{ar}}\right), 156.68(\mathrm{C}=\mathrm{N}$ cyclic), $188.99(\mathrm{C}=\mathrm{S}) \mathrm{ppm}$.

\section{1-Phenyl-2-(3-phenyl-4-thioxo-3,4-dihydroquinazolin-} 2-ylthio)ethanone (3c).

IR: $v\left(\mathrm{~cm}^{-1}\right) 1193(\mathrm{C}=\mathrm{S}), 1635\left(\mathrm{C}=\mathrm{N}_{\text {cyclic }}\right), 1728(\mathrm{C}=\mathrm{O}$ $\mathrm{Ph}), 2979\left(\mathrm{CH}_{\text {aliph. }}\right), 3088\left(\mathrm{CH}_{\text {ar. }}\right) ;{ }^{1} \mathbf{H}-\mathbf{N M R}\left(\mathrm{DMSO}-d_{6}\right): \delta$ 4.76 (s, 2H, $\left.\mathrm{CH}_{2}\right), 7.20-8.51\left(\mathrm{~m}, 14 \mathrm{H}, \mathrm{H}_{\text {ar. }}\right)$ ppm.; ${ }^{13} \mathrm{C}-$ NMR (DMSO-d $)_{6}$ (fig. 17): $\delta 43.71\left(\mathrm{CH}_{2} \mathrm{CO}\right), 126,21$, $128.20,128.77,128.93,129.90,130.06,133.47$ and $135.28\left(\mathrm{C}_{\text {ar. }}\right), 186.11\left(\mathrm{C}=\mathrm{S}_{\text {cyclic, }}\right), 193.50\left(\mathrm{C}=\mathrm{OC}_{6} \mathrm{H}_{5}\right) \mathrm{ppm}$.

Table 1: Physical and analytical data of compounds 3a-c

\begin{tabular}{|c|c|c|c|c|c|c|}
\hline \multirow{2}{*}{ Cpd } & \multirow{2}{*}{$\begin{array}{l}\text { M.P } \\
\cdot \\
\left({ }^{\circ} \mathrm{C}\right)\end{array}$} & \multirow{2}{*}{$\begin{array}{l}\text { Yiel } \\
d \\
(\%)\end{array}$} & \multirow{2}{*}{$\begin{array}{l}\text { M.F. } \\
\text { (M. wt.) }\end{array}$} & \multicolumn{3}{|c|}{$\begin{array}{l}\text { M.A. ( } \%) ; \\
\text { Calcd/Found }\end{array}$} \\
\hline & & & & $\mathrm{C} \%$ & $\mathrm{H} \%$ & N\% \\
\hline 3a & $\begin{array}{l}192- \\
4\end{array}$ & 82 & $\begin{array}{l}\mathrm{C}_{15} \mathrm{H}_{12} \mathrm{~N}_{2} \mathrm{~S}_{2} \\
1 / 10 \mathrm{H}_{2} \mathrm{O} \\
(284.4)\end{array}$ & $\begin{array}{l}63.3 \\
5 \\
62.9 \\
6\end{array}$ & $\begin{array}{l}4.2 \\
5 \\
4.4 \\
6\end{array}$ & $\begin{array}{l}9.8 \\
5 \\
9.3 \\
1\end{array}$ \\
\hline $\mathbf{3 b}$ & $\begin{array}{l}122- \\
4\end{array}$ & 75 & $\begin{array}{l}\mathrm{C}_{21} \mathrm{H}_{16} \mathrm{~N}_{2} \mathrm{~S}_{2} \\
1 / 10 \mathrm{H}_{2} \mathrm{O} \\
(360.08)\end{array}$ & $\begin{array}{l}69.9 \\
7 \\
69.6 \\
3\end{array}$ & $\begin{array}{l}4.4 \\
7 \\
4.4 \\
2\end{array}$ & $\begin{array}{l}7.7 \\
7 \\
7.7 \\
3\end{array}$ \\
\hline $3 c$ & $\begin{array}{l}181- \\
3\end{array}$ & 86 & $\begin{array}{l}\mathrm{C}_{22} \mathrm{H}_{16} \mathrm{~N}_{2} \mathrm{OS} \\
2 \\
(389.07)\end{array}$ & $\begin{array}{l}68.0 \\
1 \\
67.4 \\
8\end{array}$ & $\begin{array}{l}4.1 \\
5 \\
3.9 \\
3\end{array}$ & $\begin{array}{l}7.2 \\
1 \\
6.4 \\
5\end{array}$ \\
\hline
\end{tabular}

Reaction of compound 1 with ethyl chloroformate and/or ethyl chloroacetate. Formation of $4 \mathbf{a}, \mathbf{b}$.

Compound 1 (0.81g, $0.003 \mathrm{~mol})$ was fused in ethyl chloroformate and / or ethyl chloroacetate $(0.003 \mathrm{~mol})$ for 4-5 hr (tlc). The excess of the reagent was evaporated till dryness under vaccum. The residual solid was crystallized from ethanol to give compounds $\mathbf{4 a}$ and/or $\mathbf{4 b}$, respectively.

Ethyl-2-(3-phenyl-4-thioxo-1,2,3,4tetrahydroquinazolin-2-ylthio)formate (4a).

IR: $v\left(\mathrm{~cm}^{-1}\right) 1235(\mathrm{C}=\mathrm{S}), 1690(\mathrm{C}=\mathrm{N}), 1760(\mathrm{C}=\mathrm{O}), 2945$ $\left(\mathrm{CH}_{\text {aliph. }}\right) ;{ }^{1} \mathbf{H}-\mathbf{N M R}$ (DMSO-d $d_{6}$ (fig. 19): $\delta 1.25$ (t, $3 \mathrm{H}, J$ $\left.=3.61 \mathrm{~Hz}, \mathrm{CH}_{3}\right), 3.65\left(\mathrm{q}, 2 \mathrm{H}, J=2.96 \mathrm{~Hz}, \mathrm{CH}_{2}\right), 7.20-$ $8.55\left(\mathrm{~m}, 18 \mathrm{H}, \mathrm{H}_{\mathrm{ar}}\right.$ ) ppm.; ${ }^{13} \mathbf{C}-\mathbf{N M R}$ (DMSO- $\left.d_{6}\right): \delta 22.50$ $\left(\mathrm{CH}_{3}\right)$, 58.11 $\left(\mathrm{CH}_{2}\right), 115.58,115.93,125.20,127.90$,
$127.95,128.51,129.06,129.19,131.70,131.76,135.23$, 125.31 and $135.73\left(\mathrm{C}_{\mathrm{ar}}\right), 144.16(\mathrm{COO}), 172.81(\mathrm{C}=\mathrm{S})$, $189.77(\mathrm{C}=\mathrm{S}) \mathrm{ppm}$.

Ethyl-2-(3-phenyl-4-thioxo-1,2,3,4tetrahydroquinazolin-2-ylthio)acetate (4b).

IR: $v\left(\mathrm{~cm}^{-1}\right) 1197(\mathrm{C}=\mathrm{S}), 1610(\mathrm{C}=\mathrm{N}), 1675(\mathrm{C}=\mathrm{O}), 2930$ $\left(\mathrm{CH}_{\text {aliph. }}\right.$ ); ${ }^{1} \mathbf{H}-\mathbf{N M R}$ (DMSO- $d_{6}$ ) (fig. 22): $\delta 1.25$ (t, 3H, $J$ $\left.=4.71 \mathrm{~Hz}, \mathrm{CH}_{3}\right), 3.57\left(\mathrm{~d}, 2 \mathrm{H}, J=3.14 \mathrm{~Hz}, \mathrm{CH}_{2} \mathrm{~S}\right), 4.21(\mathrm{q}$, $\left.2 \mathrm{H}, J=2.83 \mathrm{~Hz}, \mathrm{CH}_{2} \mathrm{CO}\right), 6.20-7.85\left(\mathrm{~m}, 18 \mathrm{H}, \mathrm{H}_{\mathrm{ar} .}\right)$ ppm; ${ }^{13} \mathbf{C}-\mathbf{N M R}$ (DMSO-d $\left.d_{6}\right): \delta 14.02 \quad\left(\mathrm{CH}_{3}\right), 33.45$ $\left(\mathrm{CH}_{2} \mathrm{CO}\right), 61.45\left(\mathrm{CH}_{2}\right), 110.12,119.89,129.80,130.01$, 136.01, 137.98, 139.32, 143.32, 151.02 and $154.34\left(\mathrm{C}_{\mathrm{ar} .}\right)$, $173.02(\mathrm{C}=\mathrm{N}), 179.23(\mathrm{C}=\mathrm{O}), 203.98(\mathrm{C}=\mathrm{S}) \mathrm{ppm}$.

$\underline{\text { Reaction of compound } 1 \text { with ethyl chloroacetoacetate. }}$ Formation of 5.

To a solution of compound $1(0.81 \mathrm{~g}, 0.003 \mathrm{~mol})$ in methanol $(15 \mathrm{ml})$ and potassium hydroxide $(0.56 \mathrm{~g}, 0.01$ $\mathrm{mol})$, ethyl chloroacetoacetate $(0.49 \mathrm{~g}, 0.003 \mathrm{~mol})$ was added. The reaction mixture was stirred at $\mathrm{r}$. t. for $5 \mathrm{hr}$ (tlc). The solid product that formed was recrystallized from ethanol to give $\mathbf{5}$.

Ethyl-3-oxo-2-(3-phenyl-4-thioxo-1,2,3,4tetrahydroquinazolin-2-ylthio)-butanoate (5).

IR: $v\left(\mathrm{~cm}^{-1}\right) 1250(\mathrm{C}=\mathrm{S}), 1610(\mathrm{C}=\mathrm{N}), 1742,1665(2 \mathrm{C}=\mathrm{O})$ $2940\left(\mathrm{CH}_{\text {aliph. }}\right) ;{ }^{1} \mathbf{H}-\mathbf{N M R}$ (DMSO- $\left.d_{6}\right)$ (fig. 25): $\delta 1.64(\mathrm{t}$, $\left.3 \mathrm{H}, J=4.91 \mathrm{~Hz}, \mathrm{CH}_{3} \mathrm{CH}_{2}\right), 2.55$ (s, 3H, $\left.\mathrm{CH}_{3} \mathrm{CO}\right), 3.4$ (q, $\left.2 \mathrm{H}, J=2.53 \mathrm{~Hz}, \mathrm{CH}_{2}\right), 3.95(\mathrm{~s}, 1 \mathrm{H}, \mathrm{CH}), 7.00-7.9(\mathrm{~m}, 9 \mathrm{H}$, $\mathrm{H}_{\text {ar. }}$ ) ppm; MS: (EI) $\mathrm{m} / \mathrm{z}=398.8\left(\mathrm{M}^{+}, \mathrm{C}_{20} \mathrm{H}_{18} \mathrm{~N}_{2} \mathrm{O}_{3} \mathrm{~S}_{2}, 2.2\right.$ $\%)$.

Reaction of compound 1 with diphenyl diazomethane. Formation of 6.

Compound 1 (0.81 g, $0.003 \mathrm{~mol})$ was refluxed with diphenyl diazomethane $(0.5 \mathrm{ml}, 0.004 \mathrm{~mol})$ in anhydrous benzene $(30 \mathrm{ml})$ for $6 \mathrm{hr}(\mathrm{tlc})$. After cooling to $\mathrm{r}$. t. the formed solid product was filtered off, recrystallized from methanol, filtered, and dried to afford $\mathbf{6}$.

\section{2-(Benzhydrylthio)-3-phenylquinazoline4(3H)thione(6)}

IR: $v\left(\mathrm{~cm}^{-1}\right) 1240(\mathrm{C}=\mathrm{S}), 1690(\mathrm{C}=\mathrm{N}), 2927\left(\mathrm{CH}_{\mathrm{aliph}}\right)$, $3090\left(\mathrm{CH}_{\text {ar. }}\right){ }^{1} \mathbf{H}-\mathbf{N M R}$ (DMSO- $\left.d_{6}\right)$ (fig. 28): $\delta 3.51(\mathrm{~s}$, 1H, SCH), 6.32-8.51 (m, 19H, Har.) ppm; ${ }^{13} \mathbf{C}-\mathbf{N M R}$ $\left(\mathrm{DMSO}-d_{6}\right): \delta 55.29(\mathrm{CH}), 127.28, \quad 128.20,128.52$, 128.61, 128.94, 129.53, 129.86 and $140.07\left(\mathrm{C}_{\mathrm{ar} .}\right), 156.12$ $(\mathrm{C}=\mathrm{N}), 190.01(\mathrm{C}=\mathrm{S}) \mathrm{ppm} ; \mathrm{MS}(\mathrm{EI}) \mathrm{m} / \mathrm{z}=436.59\left(\mathrm{M}^{+}\right.$, $\mathrm{C}_{27} \mathrm{H}_{20} \mathrm{~N}_{2} \mathrm{~S}_{2}, 31.9 \%$ ).

Table2. Physical and analytical data of compounds $\mathbf{4 a , b}, \mathbf{5}$ and 6.

\begin{tabular}{|c|c|c|c|c|c|c|}
\hline \multirow{2}{*}{ Cpd } & \multirow{2}{*}{$\begin{array}{l}\text { M.P } \\
\cdot \\
\left({ }^{\circ} \mathrm{C}\right)\end{array}$} & \multirow{2}{*}{$\begin{array}{l}\text { Yiel } \\
\mathrm{d} \\
(\%)\end{array}$} & \multirow{2}{*}{$\begin{array}{l}\text { M.F. } \\
\text { (M. wt.) }\end{array}$} & \multicolumn{3}{|c|}{$\begin{array}{l}\text { M.A. ( } \%) ; \\
\text { Calcd/Found }\end{array}$} \\
\hline & & & & $\mathrm{C} \%$ & $\mathrm{H} \%$ & $\mathrm{~N} \%$ \\
\hline $\mathbf{4 a}$ & $\begin{array}{l}184 \\
-6\end{array}$ & 87 & $\begin{array}{l}\mathrm{C}_{17} \mathrm{H}_{14} \mathrm{~N}_{2} \mathrm{O}_{2} \mathrm{~S} \\
2.1 / 10 \mathrm{H}_{2} \mathrm{O}\end{array}$ & $\begin{array}{l}59.6 \\
3\end{array}$ & $\begin{array}{l}4.1 \\
2\end{array}$ & $\begin{array}{l}8.1 \\
8\end{array}$ \\
\hline
\end{tabular}




\begin{tabular}{|c|c|c|c|c|c|c|}
\hline & & & $(342.44)$ & $\begin{array}{l}59.2 \\
6\end{array}$ & $\begin{array}{l}4.0 \\
6\end{array}$ & $\begin{array}{l}8.1 \\
3\end{array}$ \\
\hline $4 b$ & $\begin{array}{l}210 \\
-12\end{array}$ & 89 & $\begin{array}{l}\mathrm{C}_{18} \mathrm{H}_{16} \mathrm{~N}_{2} \mathrm{O}_{2} \mathrm{~S} \\
2.1 / 10 \mathrm{H}_{2} \mathrm{O} \\
(356.46)\end{array}$ & $\begin{array}{l}60.6 \\
5 \\
60.2 \\
9\end{array}$ & $\begin{array}{l}4.5 \\
2 \\
4.4 \\
6\end{array}$ & $\begin{array}{l}7.8 \\
6 \\
7.8 \\
1\end{array}$ \\
\hline 5 & $\begin{array}{l}190 \\
-2\end{array}$ & 78 & $\begin{array}{l}\mathrm{C}_{20} \mathrm{H}_{18} \mathrm{~N}_{2} \mathrm{O}_{3} \mathrm{~S} \\
2.1 / 10 \mathrm{H}_{2} \mathrm{O} \\
(398.5)\end{array}$ & $\begin{array}{l}60.2 \\
8 \\
59.9 \\
5\end{array}$ & $\begin{array}{l}4.5 \\
5 \\
4.4 \\
9\end{array}$ & $\begin{array}{l}7.0 \\
3 \\
6.9 \\
9\end{array}$ \\
\hline 6 & $\begin{array}{l}196 \\
-8\end{array}$ & 85 & $\begin{array}{l}\mathrm{C}_{27} \mathrm{H}_{20} \mathrm{~N}_{2} \mathrm{~S}_{2} . \\
1 / 10 \mathrm{H}_{2} \mathrm{O} \\
(436.59)\end{array}$ & $\begin{array}{l}74.2 \\
8 \\
73.9 \\
0\end{array}$ & $\begin{array}{l}4.6 \\
2 \\
4.5 \\
6\end{array}$ & $\begin{array}{l}6.4 \\
2 \\
6.3 \\
8\end{array}$ \\
\hline
\end{tabular}

Reaction of compound 7 with a mixture of triethyl- and/or triphenyl-phosphite and aromatic aldehydes. Formation of 8a-d.

A mixture of 7 (0.98 g, $0.0028 \mathrm{~mol})$, benzaldehyde and 4clorobanzaldehyde $(0,003 \mathrm{~mol})$ and triethyl- or triphenylphosphite $(0.003 \mathrm{~mol})$ in glacial acetic acid $(30 \mathrm{ml})$ was heated at $100{ }^{\circ} \mathrm{C}$ for $4-6 \mathrm{hr}$ (tlc). The reaction mixture was concentrated to $1 / 4$ volume and poured onto ice. The solid formed was filtered off, washed by petroleum ether followed by recrystalliztion from methanol to give the amino phosphonates (8a-d), respectively. The data are listed in table 3 .

Diethyl(6,8-dibromo-4-oxo-2-thioxo-1,2dihydroquinazolin3(4H)-ylamino)- (phenyl)-methylphosphonate (8a).

IR: $v\left(\mathrm{~cm}^{-1}\right) 680(\mathrm{C}-\mathrm{Br}), 745(\mathrm{C}-\mathrm{Cl}), 1260\left(\mathrm{C}=\mathrm{S}_{\text {cyclic }}\right)$, $1310(\mathrm{P}=\mathrm{O}), 1687(\mathrm{C}=\mathrm{O}), 2849(\mathrm{CH}), 3197,3300(2 \mathrm{NH})$; ${ }^{1} \mathrm{H}-\mathrm{NMR}\left(\mathrm{DMSO}-d_{6}\right): \delta 1.22\left(\mathrm{~s}, 6 \mathrm{H}, 2 \mathrm{CH}_{3}\right), 2.52(\mathrm{~s}, 1 \mathrm{H}$, $\left.\mathrm{NH}_{\text {acyclic }}\right), 3.94(\mathrm{~s}, 1 \mathrm{H}, \mathrm{CH}), 4.15\left(\mathrm{~s}, 4 \mathrm{H}, 2 \mathrm{CH}_{2}\right), 7.42-8.05$ (m, 6H, $\left.\mathrm{H}_{\text {ar. }}\right), 8.55$ (s, 1H, $\left.\mathrm{NH}_{\text {cyclic }}\right)$ ppm. ${ }^{13} \mathbf{C}-\mathbf{N M R}$ $\left(\mathrm{DMSO}-\mathrm{d}_{6}\right): \delta 14.17\left(2 \mathrm{CH}_{3}\right), 39.29(\mathrm{CH}), 61.12\left(2 \mathrm{CH}_{2}\right)$, $126.59,127.44,129.00,129.96,130.15,130.50,135.50$ and $139.93\left(\mathrm{C}_{\mathrm{ar} .}\right), 168.20(\mathrm{C}=\mathrm{O}), 188.86(\mathrm{C}=\mathrm{S}) \mathrm{ppm}$.

Diethyl(4-chlorophenyl)(5,7-dibromo-1-oxo-3-thioxo3,4-dihydroisoquinolin-2(1H)ylamino)methylphosphonate $(8 b)$.

IR: $v\left(\mathrm{~cm}^{-1}\right) 695(\mathrm{C}-\mathrm{Br}), 1172(\mathrm{C}=\mathrm{S}), 1280(\mathrm{P}=\mathrm{O}), 1663$ $(\mathrm{C}=\mathrm{O}), 2955\left(\mathrm{CH}_{\text {aliph. }}\right), 3065\left(\mathrm{CH}_{\mathrm{ar}}\right), 3231,3410(2 \mathrm{NH})$; ${ }^{1}$ H-NMR (DMSO- $\left.d_{6}\right): \delta 1.20\left(\mathrm{~s}, 6 \mathrm{H}, 2 \mathrm{CH}_{3}\right), 2.51(\mathrm{~s}, 1 \mathrm{H}$, $\left.\mathrm{NH}_{\text {acyclic }}\right), 3.94(\mathrm{~s}, 1 \mathrm{H}, \mathrm{CH}), 4.15\left(\mathrm{~s}, 4 \mathrm{H}, 2 \mathrm{CH}_{2}\right), 7.40-8.05$ (m, 6H, $\left.\mathrm{H}_{\mathrm{ar}}\right), 8.55$ (s, 1H, $\left.\mathrm{NH}_{\text {cyclic }}\right)$ ppm.; ${ }^{13} \mathbf{C}-\mathbf{N M R}$ $\left(\mathrm{DMSO}-d_{6}\right): \delta 14.61\left(2 \mathrm{CH}_{3}\right), 39.49(\mathrm{CH}), 62.16\left(2 \mathrm{CH}_{2}\right)$, $127.71,127.84,129.82,129.94,130.24,131.63,136.11$ and $138.51\left(\mathrm{C}_{\mathrm{ar}}\right), 167,18(\mathrm{C}=\mathrm{O}), 186.14(\mathrm{C}=\mathrm{S}) \mathrm{ppm}$. Diphenyl(6,8-dibromo-4-oxo-2-thioxo-1,2dihydroquinazolin-3(4H)ylamino) (phenyl)methylphosphonate $(8 \mathrm{c})$.

IR: $v\left(\mathrm{~cm}^{-1}\right) 680(\mathrm{C}-\mathrm{Br}), 1120(\mathrm{C}=\mathrm{S}), 1260(\mathrm{P}=\mathrm{O}), 1640$ $(\mathrm{C}=\mathrm{O}), 2849(\mathrm{CH}), 3321(\mathrm{NH}) ;{ }^{1} \mathbf{H}-\mathbf{N M R}\left(\mathrm{DMSO}-d_{6}\right): \delta$ $2.50\left(\mathrm{~s}, 1 \mathrm{H}, \mathrm{NH}_{\text {acyclic }}\right), 4.01(\mathrm{~s}, 1 \mathrm{H}, \mathrm{CH}), 6.45-8.05(\mathrm{~m}$,
17H, $\left.\mathrm{H}_{\text {ar. }}\right), 8.55$ (s, 1H, NH $\left.\mathrm{Nyclic}_{\text {c }}\right)$ ppm.; ${ }^{13} \mathbf{C}-\mathbf{N M R}$ (DMSO$\left.d_{6}\right): \delta 53.55(\mathrm{CH}), 125.51,126.35,128.29,129.18,131.73$, 133.92, 134.56 and $140.73\left(2 \mathrm{Ph}, \mathrm{C}_{\mathrm{ar}}\right), 154.33$ (P-O-C), 169,22 $(\mathrm{C}=\mathrm{O}), 185.92(\mathrm{C}=\mathrm{S}) \mathrm{ppm}$.

Diphenyl(4-chlorophenyl)(5,7-dibromo-1-oxo-3thioxo3,4-dihydroisoquinolin-2(1H)ylamino)methylphosphonate $(\mathbf{8 d})$.

IR: $v\left(\mathrm{~cm}^{-1}\right) 603(\mathrm{C}-\mathrm{Br}), 692(\mathrm{C}-\mathrm{Cl}), 1185(\mathrm{C}=\mathrm{S}), 1265$ $(\mathrm{P}=\mathrm{O}), 1640(\mathrm{C}=\mathrm{O}), 2845(\mathrm{CH}), 3321(\mathrm{NH}){ }^{1}{ }^{1} \mathbf{H}-\mathbf{N M R}$ $\left(\mathrm{DMSO}-d_{6}\right): \delta 2.53\left(\mathrm{~s}, 1 \mathrm{H}, \mathrm{NH}_{\text {acyclic }}\right), 4.12(\mathrm{~s}, 1 \mathrm{H}, \mathrm{CH})$, 6.45-8.05 (m, 16H, Har. $), 8.55\left(\mathrm{~s}, 1 \mathrm{H}, \mathrm{NH}_{\text {cyclic }}\right)$ ppm.; ${ }^{13} \mathrm{C}-$ NMR (DMSO- $\left.d_{6}\right): \delta 53.15(\mathrm{CH}), 127.00,128.84,129.34$, 129.98, 130.33, 131.91, 136.23 and $138.85\left(\mathrm{C}_{\mathrm{ar} .}\right), 152.10$ (P-O-C), $169.46(\mathrm{C}=\mathrm{O}), 189.09(\mathrm{C}=\mathrm{S}) \mathrm{ppm}$.

Table 3. Physical and analytical data of compounds 8a-d.

\begin{tabular}{|c|c|c|c|c|c|c|}
\hline \multirow[t]{2}{*}{ Cpd } & \multirow{2}{*}{$\begin{array}{l}\text { M.P } \\
\cdot \\
\left({ }^{\circ} \mathrm{C}\right)\end{array}$} & \multirow{2}{*}{$\begin{array}{l}\text { Yiel } \\
d \\
(\%)\end{array}$} & \multirow{2}{*}{$\begin{array}{l}\text { M.F. } \\
\text { (M. wt.) }\end{array}$} & \multicolumn{3}{|c|}{$\begin{array}{l}\text { M.A. (\%); } \\
\text { Calcd/Found }\end{array}$} \\
\hline & & & & $\mathrm{C} \%$ & $\mathrm{H} \%$ & $\mathrm{~N} \%$ \\
\hline 8a & $\begin{array}{l}215- \\
17\end{array}$ & 87 & $\begin{array}{l}\mathrm{C}_{19} \mathrm{H}_{19} \mathrm{Br}_{2} \mathrm{ClN}_{3} \mathrm{O}_{4} \mathrm{PS} .1 / 5 \mathrm{H}_{2} \mathrm{O} \\
(611.67)\end{array}$ & $\begin{array}{l}37.3 \\
1 \\
37.0 \\
5\end{array}$ & $\begin{array}{l}3.1 \\
3 \\
3.0 \\
8\end{array}$ & $\begin{array}{l}6.8 \\
7 \\
6.8 \\
2\end{array}$ \\
\hline $\mathbf{8 b}$ & $\begin{array}{l}195- \\
17\end{array}$ & 89 & $\begin{array}{l}\mathrm{C}_{19} \mathrm{H}_{20} \mathrm{Br}_{2} \mathrm{~N}_{3} \mathrm{O}_{4} \mathrm{PS} .1 / 25 \mathrm{MeOH} .1 / 20 \mathrm{H}_{2} \\
\mathrm{O} \\
(437.11)\end{array}$ & $\begin{array}{l}39.5 \\
3 \\
39.5 \\
1\end{array}$ & $\begin{array}{l}3.4 \\
9 \\
3.3 \\
7\end{array}$ & $\begin{array}{l}7.2 \\
8 \\
\\
5.9 \\
6\end{array}$ \\
\hline $8 \mathrm{c}$ & $\begin{array}{l}220- \\
22\end{array}$ & 80 & $\begin{array}{l}\mathrm{C}_{27} \mathrm{H}_{20} \mathrm{Br}_{2} \mathrm{~N}_{3} \mathrm{O}_{4} \mathrm{PS} .1 / 20 \mathrm{H}_{2} \mathrm{O} \\
(673.31)\end{array}$ & $\begin{array}{l}48.1 \\
6 \\
48.0 \\
5\end{array}$ & $\begin{array}{l}2.9 \\
9 \\
2.9 \\
6\end{array}$ & $\begin{array}{l}6.2 \\
4 \\
6.2 \\
2\end{array}$ \\
\hline $8 d$ & $\begin{array}{l}189- \\
91\end{array}$ & 77 & $\begin{array}{l}\mathrm{C}_{27} \mathrm{H}_{19} \mathrm{Br}_{2} \mathrm{ClN}_{3} \mathrm{O}_{4} \mathrm{PS} .1 / 20 \mathrm{H}_{2} \mathrm{O} \\
(707.76)\end{array}$ & $\begin{array}{l}45.8 \\
2 \\
45.7 \\
2\end{array}$ & $\begin{array}{l}2.7 \\
1 \\
2.6 \\
8\end{array}$ & $\begin{array}{l}5.9 \\
4 \\
5.9 \\
2\end{array}$ \\
\hline
\end{tabular}

Reaction of compound 7 with 4-aminobenzoic acid. Formation of 9.

A mixture of $7(0.7 \mathrm{~g}, 0.002 \mathrm{~mol})$ and 4-aminobenzoic acid was heated in boiling phosphorus oxychloride $(10 \mathrm{ml})$ for $8 \mathrm{hr}$ (tlc.). The reaction mixture was cooled to r. t. and neutralized by $\mathrm{NaOH}(20 \%)$. The solid formed was filtered off, recrystallized from ethanol to give 9, yield $77 \%$, m. p. $180^{\circ} \mathrm{C}$.

2-(4-Aminophenyl)-7,9-dibromo-10,10a-dihydro[1,3,4]thiadiazolo[2,3-b]quina-zolin-5-one (9)

IR: $v\left(\mathrm{~cm}^{-1}\right) 695(\mathrm{C}-\mathrm{Br}), 1617(\mathrm{C}=\mathrm{N}), 1655(\mathrm{C}=\mathrm{O}$, cyclic $)$, $3338\left(\mathrm{NH}_{2}\right.$ sym $), 3460\left(\mathrm{NH}_{2}\right.$ asym $) .{ }^{1} \mathbf{H}-\mathrm{NMR}$ (DMSO- $\left.d_{6}\right): \delta$ 4.09 (s, 2H, $\left.\mathrm{NH}_{2}\right), 6.65-8.55\left(\mathrm{~m}, 6 \mathrm{H}, \mathrm{H}_{\text {ar. }}\right)$ ppm.; ${ }^{13} \mathrm{C}-$ NMR (DMSO- $\left.d_{6}\right): \delta 115.14,118.64,128.55,128.65$, $129.89,129.96,131.70,133.41$ and $138.81\left(\mathrm{C}_{\mathrm{ar}}\right), 153.15$ $(\mathrm{C}=\mathrm{C}), 164.32(\mathrm{C}=\mathrm{O}), 170,31(\mathrm{C}=\mathrm{N}) \mathrm{ppm}$.

Analysis for $\mathrm{C}_{15} \mathrm{H}_{8} \mathrm{Br}_{2} \mathrm{~N}_{4} \mathrm{OS}$. 1/20 $\mathrm{H}_{2} \mathrm{O}$ (M.wt.452)

Calcd: C, 39.85\%; H, 1.78\%; N, 12.39\%.. Found: $39.12 \% ; \quad 1.57 \% ; \quad 11.75 \%$. 
Reaction of compound 7 with triethylorthoformate. Formation of 10.

A mixture of compound $7(0.7 \mathrm{~g}, 0.002 \mathrm{~mol})$ and triethylorthoformate $(0.43 \mathrm{~g}, 0.003 \mathrm{~mol})$ in glacial acetic acid $(25 \mathrm{ml})$ was refluxed for $3 \mathrm{hr}(\mathrm{tlc})$. The reaction mixture was cooled to r.t. The solid product that formed was filtered off, recrystallized from ethanol and dried to give 10. yield $76 \%$, m. p. $192{ }^{\circ} \mathrm{C}$.Ethyl N-6,8-dibromo-4-oxo-2-thioxo-1,2 dihydroquinazolin-3(4H)-ylformimidate (10)

IR: $v \quad\left(\mathrm{~cm}^{-1}\right) \quad 670 \quad(\mathrm{C}-\mathrm{Br}), \quad 1630 \quad(\mathrm{C}=\mathrm{N}), \quad 1740$ $\left(\mathrm{C}=\mathrm{O}_{\text {cyclic }}\right), 1180\left(\mathrm{C}=\mathrm{S}_{\text {cyclic }}\right), 2950(\mathrm{CH}), 3498(\mathrm{NH})$; ${ }^{1}$ H-NMR (DMSO- $\left.d_{6}\right): \delta 1.61(\mathrm{t}, 3 \mathrm{H}, J=4.11 \mathrm{~Hz}$, $\left.\mathrm{CH}_{3}\right), 4.10$ (q, 2H, $\left.J=2.17 \mathrm{~Hz}, \mathrm{CH}_{2}\right), 1663\left(\mathrm{C}=\mathrm{S}_{\text {cyclic }}\right)$ ppm.; ${ }^{13}$ C-NMR (DMSO-d $\left.d_{6}\right): \delta 14.19\left(\mathrm{CH}_{3}\right), 61.52$ $\left(\mathrm{CH}_{2}\right), 126.59,127.44,129.00,130.15,135.50$ and $140.91\left(\mathrm{C}_{\text {ar. }}\right), 155.61(\mathrm{C}=\mathrm{N}), 167.85(\mathrm{C}=\mathrm{O}), 186,72$ $(\mathrm{C}=\mathrm{S}) \mathrm{ppm}$.

Analysis for $\mathrm{C}_{11} \mathrm{H}_{9} \mathrm{Br}_{2} \mathrm{~N}_{3} \mathrm{O}_{2} \mathrm{~S}$. 1/25EtOH. $1 / 20 \mathrm{H}_{2} \mathrm{O}$ (407.08)

Calcd: C, 32.45\%; H, 2.23\%; N, 10.32\%. Found: $32.35 \%$; $2.20 \% ; \quad 10.29 \%$.

Reaction of compound 7 with 4-benzylidene-2phenyloxazol-5(4H)-one. Formation of 11

A mixture of compound $7(0.7 \mathrm{~g}, 0.002 \mathrm{~mol})$ and 4benzylidene-2-phenyloxazol-5(4H)-one (a) $(0.5 \mathrm{~g}, 0.002$ mol) was boiled in glacial acetic acid for $5 \mathrm{hr}$ (tlc.), The reaction mixture was cooled to r.t. The solid product that formed was filtered off, recrystallized from ethanol and dried to give 11. yield $60 \%$, m. p. $210^{\circ} \mathrm{C}$.

3-(4-Benzylidene-5-oxo-2-phenyl-4,5-dihydroimidazol1-yl)-6,8-dibromo-2-thioxo-1,2,3,8atetrahydroquinazolin-4(4aH)-one (11).

IR: $v\left(\mathrm{~cm}^{-1}\right) 705(\mathrm{C}-\mathrm{Br}), 1160\left(\mathrm{C}=\mathrm{S}_{\mathrm{cyclic}}\right), 1590(\mathrm{C}=\mathrm{N})$, $1610 \quad(\mathrm{C}=\mathrm{O}-\mathrm{N}), \quad 1665 \quad\left(\mathrm{C}=\mathrm{O}_{\text {cyclic }}\right), \quad 2970 \quad(\mathrm{CH}), 3490$ $(\mathrm{NH}) ;{ }^{1} \mathbf{H}-\mathrm{NMR}\left(\mathrm{DMSO}-d_{6}\right): \delta 4.22(\mathrm{~s}, 1 \mathrm{H}, \mathrm{NH}), 7.19(\mathrm{~s}$, $1 \mathrm{H}, \mathrm{CH}), 7.20-8.54\left(\mathrm{~m}, 12 \mathrm{H}, \mathrm{H}_{\mathrm{ar} .}\right)$ ppm.; ${ }^{13} \mathbf{C}-\mathbf{N M R}$ $\left(\mathrm{DMSO}-d_{6}\right): \delta 115.58(\mathrm{CH}), 125.20,127.90,127.95$, 128.51, 129.06, 129.19, 131.70, 135.23, 135.31, 135.73 $\left(\mathrm{C}_{\text {ar. }}\right), 163.01\left(\mathrm{C}=\mathrm{O}_{\text {cyclic }}\right), 172.81(\mathrm{C}=\mathrm{O}), 189.77(\mathrm{C}=\mathrm{S})$ ppm.; MS (EI) m/z $=\left(582.27, \mathrm{C}_{24} \mathrm{H}_{14} \mathrm{Br}_{2} \mathrm{~N}_{4} \mathrm{O}_{2} \mathrm{~S}, 10 \%\right)$.

Analysis for $\mathrm{C}_{24} \mathrm{H}_{14} \mathrm{Br}_{2} \mathrm{~N}_{4} \mathrm{O}_{2} \mathrm{~S} .1 / 20 \mathrm{H}_{2} \mathrm{O}$ (582.27)

Calcd: C, 49.51\%; H, 2.42\%; N, 9.62\%. Found: $49.38 \% ; \quad 2.40 \% ; \quad 9.60 \%$.

Reaction of compound 7 with 4-chlorophenyl isocyanate and/or phenyl isothyocyanate. Formation of $\underline{12}$ and 14.

To a solution of compound $7(0.35 \mathrm{~g}, 0.001 \mathrm{~mol})$ in anhydrous pyridine $(25 \mathrm{ml})$ was added 4-chlorophenyl isocyanate and / or phenyl isothyocyanate $(0.006 \mathrm{~mol})$. The reaction mixture was refluxed for 4-5 hr (tlc). The solvent was evaporated to dryness under vacuum, and the residual solid was recrystallized from methanol and dried to give 12 and / or 14, respectively.

1-(4-Chlorophenyl)-3-(6,8-dibromo-4-oxo-2-thioxo1,2-dihydroquinazolin-3(4H)-yl)urea (12).

IR: $v\left(\mathrm{~cm}^{-1}\right) 1190 \quad\left(\mathrm{C}=\mathrm{S}_{\text {cyclic }}\right), 1610 \quad\left(\mathrm{C}=\mathrm{O}_{\text {cyclic }}\right), 1660$ $(\mathrm{C}=\mathrm{O}-\mathrm{NH})$, , $2961(\mathrm{CH}), 3450(\mathrm{NH}){ }^{1}{ }^{1} \mathbf{H}-\mathbf{N M R}$ (DMSO$\left.d_{6}\right): \delta 7.30-8.21\left(\mathrm{~m}, 7 \mathrm{H}, \mathrm{H}_{\text {ar. }}\right), 8.81\left(\mathrm{~s}, 1 \mathrm{H}, \mathrm{NH}_{\text {cyclic. }}\right) 11.72$

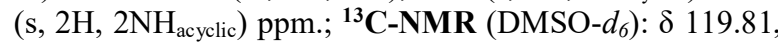
$128.61,128.87,129.40,130.96,137.78,138.54$ and $139.02\left(\mathrm{C}_{\mathrm{ar}}\right), 149.80(\mathrm{C}=\mathrm{O}), 161.08\left(\mathrm{C}=\mathrm{O}_{\text {cyclic }}\right), 179.01$ $(\mathrm{C}=\mathrm{S}) \mathrm{ppm}$.

7,9-Dibromo-2-(phenylimino)-2,3dihydro[1,3,4]thiadiazolo[2,3-b]quinazolin-5-one (14).

IR: $v(\mathrm{~cm}-1) 678(\mathrm{C}-\mathrm{Br}), 1610(\mathrm{C}=\mathrm{N}), 1690(\mathrm{C}=\mathrm{O}), 3450$ $(\mathrm{NH}) ;{ }^{1} \mathrm{H}-\mathrm{NMR}\left(\mathrm{DMSO}-d_{6}\right): \delta 2.41(\mathrm{NH}), 7.45-8.25(\mathrm{~m}$, 7H, $\left.\mathrm{H}_{\text {ar. }}\right)$ ppm. ${ }^{13} \mathbf{C}-\mathbf{N M R}\left(\mathrm{DMSO}-d_{6}\right): \delta 125.93,126.02$, $127.24,128.34,128.41,129.26,129.31,129.40$ and $137.25\left(\mathrm{C}_{\mathrm{ar}}\right), 147.17(\mathrm{C}=\mathrm{N}), 156.86(\mathrm{C}=\mathrm{O}), 160.66(\mathrm{C}-\mathrm{S})$ ppm.

Table 4. Physical and analytical data of compounds 12 and 14.

\begin{tabular}{|c|c|c|c|c|c|c|}
\hline \multirow[t]{2}{*}{ Cpd } & \multirow{2}{*}{$\begin{array}{l}\text { M.P } \\
\cdot \\
\left({ }^{\circ} \mathrm{C}\right)\end{array}$} & \multirow{2}{*}{$\begin{array}{l}\text { Yiel } \\
\text { d } \\
(\%)\end{array}$} & \multirow{2}{*}{$\begin{array}{l}\text { M.F. } \\
\text { (M. wt.) }\end{array}$} & \multicolumn{3}{|c|}{$\begin{array}{l}\text { M.A. ( \%); } \\
\text { Calcd/Found }\end{array}$} \\
\hline & & & & $\mathrm{C} \%$ & $\mathrm{H} \%$ & $\mathrm{~N} \%$ \\
\hline 12 & $\begin{array}{l}192 \\
-4\end{array}$ & 57 & $\begin{array}{l}\mathrm{C}_{15} \mathrm{H}_{9} \mathrm{Br}_{2} \mathrm{ClN}_{4} \mathrm{O}_{2} \\
\text { S. } 1 / 20 \mathrm{H}_{2} \mathrm{O} \\
(504.58)\end{array}$ & $\begin{array}{l}35.7 \\
0 \\
35.6 \\
0\end{array}$ & $\begin{array}{l}1.8 \\
0 \\
1.7 \\
8\end{array}$ & $\begin{array}{l}11.1 \\
0 \\
11.0 \\
7\end{array}$ \\
\hline 14 & $\begin{array}{l}122 \\
-4\end{array}$ & 71 & $\begin{array}{l}\mathrm{C}_{15} \mathrm{H}_{8} \mathrm{Br}_{2} \mathrm{~N}_{4} \mathrm{OS} \\
1 / 20 \mathrm{H}_{2} \mathrm{O} \\
(452.12)\end{array}$ & $\begin{array}{l}39.8 \\
5 \\
39.7 \\
3\end{array}$ & $\begin{array}{l}1.7 \\
8 \\
1.7 \\
6\end{array}$ & $\begin{array}{l}12.3 \\
9 \\
12.3 \\
6\end{array}$ \\
\hline
\end{tabular}

Reaction of compound 7 with chloroacetaldehyde. Formation of 15

A mixture of compound $7(0.35 \mathrm{~g}, 0.001 \mathrm{~mol})$ and chloroacetaldehyde $(0.15 \mathrm{~g}, 0.002 \mathrm{~mol})$ in ethanol $(20 \mathrm{ml})$ was refluxed for $3 \mathrm{hr}(\mathrm{tlc})$. The reaction mixture was cooled to r.t.. The solid product that formed was filtered off, recrystallized from ethanol and dried to give 15. yield $88 \%$, m. p. $185^{\circ} \mathrm{C}$.

2-(3-Amino-6,8-dibromo-4-oxo-1,2,3,4tetrahydroquinazolin-2-ylthio)acetaldehyde (15).

IR: $v\left(\mathrm{~cm}^{-1}\right) 730(\mathrm{C}-\mathrm{Br}), 1560(\mathrm{C}=\mathrm{N}), 1620\left(\mathrm{C}=\mathrm{O}_{\text {cyclic }}\right)$, $1690(\mathrm{C}=\mathrm{O}), 2850\left(\mathrm{CH}_{\mathrm{ar}}\right), 3300\left(\mathrm{NH}_{2} \mathrm{sym}\right), 3490\left(\mathrm{NH}_{2}\right.$ asym); ${ }^{1} \mathrm{H}-\mathrm{NMR}$ (DMSO- $\left.d_{6}\right): \delta 2.50\left(\mathrm{~s}, 2 \mathrm{H}, \mathrm{NH}_{2}\right), 4.43(\mathrm{~s}$, $\left.2 \mathrm{H}, \mathrm{CH}_{2}\right), 8.15-8.4\left(\mathrm{dd}, 2 \mathrm{H}, J=1.95 \mathrm{~Hz}, \mathrm{H}_{\mathrm{ar}}\right) 9.70$ (s, $1 \mathrm{H}, \mathrm{CH}) \mathrm{ppm}$.

Analysis for $\mathrm{C}_{10} \mathrm{H}_{7} \mathrm{Br}_{2} \mathrm{~N}_{3} \mathrm{O}_{2}$ S. $1 / 20 \mathrm{H}_{2} \mathrm{O}$ (393.05)

Calcd: C, 30.56\%; H, 1.80\%; N, 10.69\%. Found: $30.46 \% ; \quad 1.77 \% ; \quad 10.66 \%$. 


\section{Reaction of 7 with some Sultones. Formation of 18a,b.}

Compound $7(0.35 \mathrm{~g}, 0.001 \mathrm{~mol})$ was fused with $1,3-$ propane- and/or 1,4-butane-sultone $(0.002 \mathrm{~mol})$ at $180^{\circ} \mathrm{C}$ for 4-6 $\mathrm{hr}(\mathrm{tlc})$. The residual solid was recrystallized from ethanol and afforded the corresponding sultams 18a and $\mathbf{1 8 b}$, respectively.

\section{6,8-Dibromo-2-thioxo-2,3dihydroquinazolinoylpr- opane -1,3-sultam (18a).}

IR: $v\left(\mathrm{~cm}^{-1}\right) 685(\mathrm{C}-\mathrm{Br}), 1120(\mathrm{C}=\mathrm{S}), 1151-1360$ $\left(\mathrm{SO}_{2}\right), \quad 1710 \quad\left(\mathrm{C}=\mathrm{O}_{\text {cyclic }}\right), 3390 \quad(\mathrm{NH}) ;{ }^{1} \mathbf{H}-\mathbf{N M R}$ $\left(\mathrm{DMSO}-d_{6}\right): \delta 1.20\left(\mathrm{~m}, 2 \mathrm{H}, \mathrm{CH}_{2}\right), 3.01(\mathrm{~d}, 2 \mathrm{H}, J=$ $\left.2.01 \mathrm{~Hz}, \mathrm{CH}_{2}\right), 3.20\left(\mathrm{~d}, 2 \mathrm{H}, J=2.21 \mathrm{~Hz}, \mathrm{CH}_{2}\right), 4.15$ (NH), $7.80-8.51$ (dd, $\left.2 \mathrm{H}, J=2.75 \mathrm{~Hz}, \mathrm{H}_{\mathrm{ar}}\right)$ ppm. ; ${ }^{13}$ C-NMR (DMSO- $\left.d_{6}\right): \delta 14.17\left(\mathrm{CH}_{2}\right), 39.29\left(\mathrm{CH}_{2}\right)$, $61.12\left(\mathrm{CH}_{2}\right), 126.59,127.49,129.00,130.15,139.93$ $\left(\mathrm{C}_{\mathrm{ar}}\right), 168.20(\mathrm{C}=\mathrm{O}), 188.86(\mathrm{C}=\mathrm{S}) \mathrm{ppm}$.

\section{6,8-Dibromo-2-thioxo-2,3dihydroquinazolinoylb- utane-1,4-sultam (18b).}

IR: $v\left(\mathrm{~cm}^{-1}\right) 690(\mathrm{C}-\mathrm{Br}), 1120(\mathrm{C}=\mathrm{S}), 1156-1350\left(\mathrm{SO}_{2}\right)$, $1670\left(\mathrm{C}=\mathrm{O}_{\text {cyclic }}\right), 3400(\mathrm{NH}) ;{ }^{1} \mathrm{H}-\mathrm{NMR}$ (DMSO- $\left.d_{6}\right): \delta$ $1.21\left(\mathrm{~m}, 2 \mathrm{H}, \mathrm{CH}_{2}\right), 2.01\left(\mathrm{~m}, 2 \mathrm{H}, \mathrm{CH}_{2}\right), 2.95(\mathrm{~d}, 2 \mathrm{H}, J=$ $\left.2.14 \mathrm{~Hz}, \mathrm{CH}_{2}\right), 3.15\left(\mathrm{~d}, 2 \mathrm{H}, J=2.71 \mathrm{~Hz}, \mathrm{CH}_{2}\right), 4.70(\mathrm{NH})$, $7.90-8.53$ (dd, $\left.2 \mathrm{H}, J=2.99 \mathrm{~Hz}, \mathrm{H}_{\text {ar. }}\right)$ ppm.; ${ }^{13} \mathbf{C}-\mathbf{N M R}$ (DMSO- $\left.d_{6}\right): \delta 19.17\left(\mathrm{CH}_{2}\right), 21.29\left(\mathrm{CH}_{2}\right), 47.51\left(\mathrm{CH}_{2}\right)$, $50.51\left(\mathrm{CH}_{2}\right), 126.69,129.96,130.15,135.35,139.93$ $\left(\mathrm{C}_{\mathrm{ar}}\right), 160.27(\mathrm{C}=\mathrm{O}), 186.45(\mathrm{C}=\mathrm{S}) \mathrm{ppm}$.

Table 5. Physical and analytical data of compounds 18a,b.

\begin{tabular}{|c|c|c|c|c|c|c|}
\hline \multirow[t]{2}{*}{ Cpd } & \multirow{2}{*}{$\begin{array}{l}\text { M.P } \\
\cdot \\
\left({ }^{\circ} \mathrm{C}\right)\end{array}$} & \multirow{2}{*}{$\begin{array}{l}\text { Yiel } \\
\text { d } \\
(\%)\end{array}$} & \multirow{2}{*}{$\begin{array}{l}\text { M.F. } \\
\text { (M. wt.) }\end{array}$} & \multicolumn{3}{|c|}{$\begin{array}{l}\text { M.A. (\%); } \\
\text { Calcd/Found }\end{array}$} \\
\hline & & & & $\mathrm{C} \%$ & $\mathrm{H} \%$ & N\% \\
\hline $18 \mathrm{a}$ & $\begin{array}{l}192- \\
4\end{array}$ & 61 & $\begin{array}{l}\mathrm{C}_{11} \mathrm{H}_{9} \mathrm{Br}_{2} \mathrm{~N}_{3} \mathrm{O}_{3} \mathrm{~S}_{2} \\
.1 / 20 \mathrm{H}_{2} \mathrm{O} \\
\quad(455.15)\end{array}$ & $\begin{array}{l}29.0 \\
3 \\
28.9 \\
4\end{array}$ & $\begin{array}{l}1.9 \\
9 \\
1.9 \\
7\end{array}$ & $\begin{array}{l}9.2 \\
3 \\
9.2 \\
0\end{array}$ \\
\hline $18 b$ & $\begin{array}{l}122- \\
4\end{array}$ & 89 & $\begin{array}{c}\mathrm{C}_{12} \mathrm{H}_{11} \mathrm{Br}_{2} \mathrm{~N}_{3} \mathrm{O}_{3} \mathrm{~S} \\
2.1 / 20 \mathrm{H}_{2} \mathrm{O} \\
\qquad \\
(469.17)\end{array}$ & $\begin{array}{l}30.7 \\
2 \\
30.6 \\
3\end{array}$ & $\begin{array}{l}2.3 \\
6 \\
2.3 \\
4\end{array}$ & $\begin{array}{l}8.9 \\
6 \\
8.9 \\
3\end{array}$ \\
\hline
\end{tabular}

\section{BIOLOGICAL ACTIVITY}

Antimicrobial activity of some selected compounds (4a and 5) were determined against Escherichia coli (NCIM2065) as gram-negative bacteria, S. ureus as grampositive bacteria and Candida albicans as fungi. The inhibition zones were measured in triplicates by standard methods using Cut plug method. ${ }^{18,19}$ It was found compound $\mathbf{5}$ exhibited the highest inhibition zone against E. coli.

\section{Conclusion}

In this work. It is reported the preparation of some new quinazoline-4-ones derivatives for testing their biological activity bactericides. The results indicated that some of them showed activity against different types of bacteria.

\section{REFERENCES}

[1] A. A. El-Barbary, A. Z. Abou-El-Ezz, A. M. Sharaf, Phosphorus, Sulfur and

Silicon, 181, 1895 (2006).

[2] A. A. El-Barbary and R. S. Azazy, Phosphorus, Sulfur and Silicon, Submitted.

[3] A. A. El-Barbary and H. El-Kabes., Reactivity of some quinazolone derivatives, Un published work.

[4] V. J. Ram, R. C. Srimal, D. S. Kushwaha and L. Mishra, J. Prakt. Chem.,323, 629 (1990).

[5] A. A. Bekhit, N. S. Habbib and A. El. Bekhit, Boll. Chim. Farm., 140, 297 (2001).

[6] J. B. Jiang, D. P. Hesson, B. A Dusak, D. L. Dexter and G. T. Kang, J. Med. Chem., 33, 1721 (1990).

[7] S. E. Lopez, M. E. Rosales, E. C. Canelon, A. E. Valverode, R. C. Narvaez, J. E. Charris, F. A. Giannini, R. D. Enriz, M. Carrasco and S. Zacchino, Heterocycl. Commun., 7, 473 (2000).

[8] A. O. Farghaly and A. M. Moharram, Boll. Chim. Farm., 138, 280 (1999).

[9] C. O. Usifoh and G. K. E. Scriba, Arch. Pharm.(Weinheim.), 333, 261 (2000).

[10] J. Tani, Y. Yamada, T. Oine, T. Ochiai, R. Ishida and I. Inoue, J. Med.Chem., 22, 5 (1979).

[11] S. Plescia, G. Daiolone, L. Ceraulo, M. L. Bajardr and R. Reina, Farmaco ED. Sci., 39, 120 (1984).

[12] P. Singh, J. Ind. Chem. Soc. 55, 801 (1978).

[13] T. Ochiai and R. Ishida, Jpn. J. Pharmacol., 31, 491 (1981).

[14] D. Raffa, G. Daidone, D. Schillaci, B. Maggio and F. Plescia, Pharmazie., 54, 251 (1999).

[15] K. Waissera, J. Gregora, H. Dostála, J. Kuneša, L.

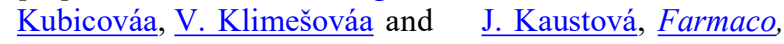
56, (803) 2001.

[16] A. A. El-Barbary, A. Z. Abou-El-Ezz, A. A. AbedKader, M. El-Daly and C. (2004)

Nielsen, Phosphorus, Sulfur and Silicon, 179, 1497

[17] L.I. Smith and K. L. Howard, Org. Synth., Coll. Vol., 351 (1955).

[18] R. Mala and M. Sarojini, Cell and Tissue Res., 9, 1951 (2009).

[19] J. Andrews, Antim. Chem., 48, 5-16 (2001). 


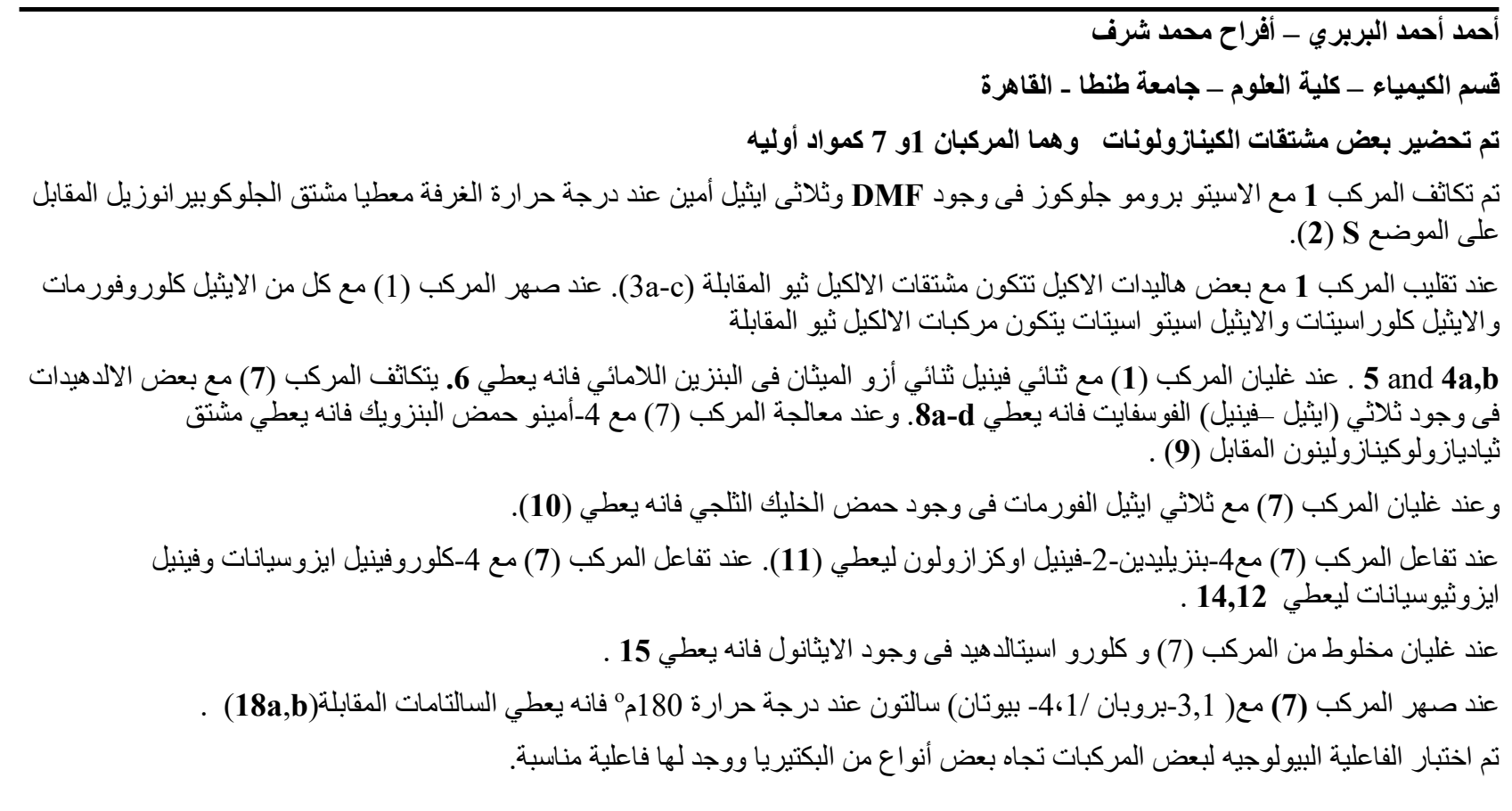


